\title{
A First Approach to a Proposal of a Soft Robotic Link Acting as a Neck
}

\author{
Luis Nagua, Jorge Muñoz, Concepción A. Monje and Carlos Balaguer \\ Systems Engineering and Automation Department, RoboticsLab \\ University Carlos III of Madrid \\ Madrid, Spain \\ lnagua@ing.uc3m.es, jmyanezb.ing@uc3m.es, cmonje@ing.uc3m.es, balaguer@ing.uc3m.es
}

\begin{abstract}
The purpose of this paper is to design a soft robotic neck prototype with two Degrees of Freedom (DOF) and propose a control system based on a fractional order PD controller (FPD). The neck will be able to perform movements of flexion, extension and lateral bending. To achieve these movements, the design is made based on a cable-driven mechanism, with components easy to manufacture in a 3D printer. Simulations are performed to validate the feasibility of the developed parallel robot prototype and the robustness of the proposed control scheme to mass changes at the tip.
\end{abstract}

Keywords: Soft robotics neck, Cable-Driven Parallel Mechanisms (CDPM), Fractional order control, Robust control, Neck prototype.

\section{Introduction}

Nowadays, there exists a new trend on biologically inspired robots with "soft" elements that are able to perform tasks which are not available to robots with rigid limbs. In the case of humanoid robotics, a robot with soft links has the following main advantages: a) simplicity of design, favoring an under-actuated architecture; b) accessibility and adaptability to complex environments; and c) safer interaction with the human and the environment.

Focusing on the neck element, there are several humanoid neck mechanisms developed by different researchers. They can be divided into two categories, i.e. serial necks and parallel necks.

The neck in series is very used due to its easy control, since each DOF is operated independently. Robotic necks in series such as HRP-4 [1] and Honda ASIMO-2002 [2] have two DOF (pitch, yaw). The four-bar robotic neck in [3] also has two DOF. There are also designs with three DOF, for example Albert HUBO [3], Dav [4] and the final design of iCub [5].

Parallel robot necks are based in general on a parallel manipulator, which consists of a mobile plat- form, a fixed base, several identical active chains and a passive backbone, if necessary. This type of mechanism is interesting for the following reasons: the number of actuators is minimal; the number of sensors necessary for the closed-loop control of the mechanism is also minimal; when the actuators are locked, the manipulator remains in its position, which is an important safety aspect for certain applications, such as medical robotics. The SAYA head has a structure composed of a central spring and several pneumatic artificial muscles [6].

In this paper a soft robotic neck is proposed based on a cable-driven parallel mechanism. It is the purpose for this soft link to be used interchangeably in various limbs of the humanoid robot, like arms, neck and spine, under the constraints of scalability, controllability of its stiffness and integration. The first step towards this goal is the design and performance analysis of a prototype of soft link working as a neck, studying its mathematical model and proposing a control system that guarantees the performance robustness to mass changes at the tip.

Due to the neck design and its elastic behavior, the actuators will have different loads at every different position, going through a varying load path for every movement. In this situation, a robust controller will be needed, and a fractional control approach is considered.

The fractional order controllers receiving the most attention in the last decades are the Fractional Proportional Integral Derivative $\left(\mathrm{PI}^{\lambda} \mathrm{D}^{\mu}\right)$ controllers, formulated for the first time by Podlubny [7] and studied in works such as [8], [9], or [10].

A remarkable number of articles, specially when the subject involves motion control, focus on the derivative control, leaving the integrator out. This control scheme has the advantage of using the position sensor as an integrator, which simplifies the controller, while the steady state error is still cancelled, making the integral part unnecessary, even undesired. The first to use this kind of fractional controller was Dorcak in [11], and later in [12] or [13], with application to the control of the joints of a robot. Similar approaches have been proposed 
in [14], [15] and [16].

There are different tuning approaches, based on different techniques. Many of them are based on the numeric solution of nonlinear equation systems [10], [12] and [13]. Other approaches based on optimization methods can be found in the literature, such as Particle Swarm Optimization algorithm (PSO) [9], [15], Artificial Bee Colony algorithm (ABC) [17], [18], Firefly Algorithm (FA) [19], or Differential Evolution method (DE) [20]. A comparative study for optimization algorithms applied to fractional controllers can be found in [21].

A novel method for the tuning of a Fractional order PD (FPD) controller is presented in this paper. Differently from other tuning methods in the literature based on numeric solutions of nonlinear equation systems or optimization, our proposal has a graphical nature, and allows the tuning of the controller in a very intuitive way through very simple and straightforward steps that do not require computational efforts.

The paper is organized as follows. Section II introduces the soft neck proposal and its mathematical model. Section III presents the control problem and proposes a novel tuning method for an FPD that improves the robustness of the system to mass changes at the neck tip. Section IV discusses the simulation results obtained from the control of the neck using a PD and an FPD controller. Finally, Section V outlines the main conclusions of the work.

\section{Robotic Neck}

The prototype proposed in this paper is the result of research on robot necks, mainly in the configuration of parallel robots for the advantages of [22]. The motions of the human neck include flexion / extension (pitch), vertical rotation (yaw) and lateral flexion (roll) [23], as shown in Fig. 1. The flexion movement with a range of $50^{\circ}$, while the extension has a range of $57.5^{\circ}$, ensures that the head is tilted backwards. The vertical rotation of the neck is described as the rotation of the head to the right and to the left with a rotation range of $70^{\circ}$. The lateral flexion allows the head to bend towards the shoulder, with a maximum angle of $45^{\circ}$.

The Cable Drive Parallel mechanism (CDPM) shown in Fig. 2(a) consists of: a fixed base, a moving platform, three flexible cables with negligible mas and a compression spring. The cables are driven by a servomotor each, as shown in the figure. The coordinate frame $O X Y Z$ is attached to the fixed base and the $\mathrm{Y}$-axis is along $O A_{1}$. The

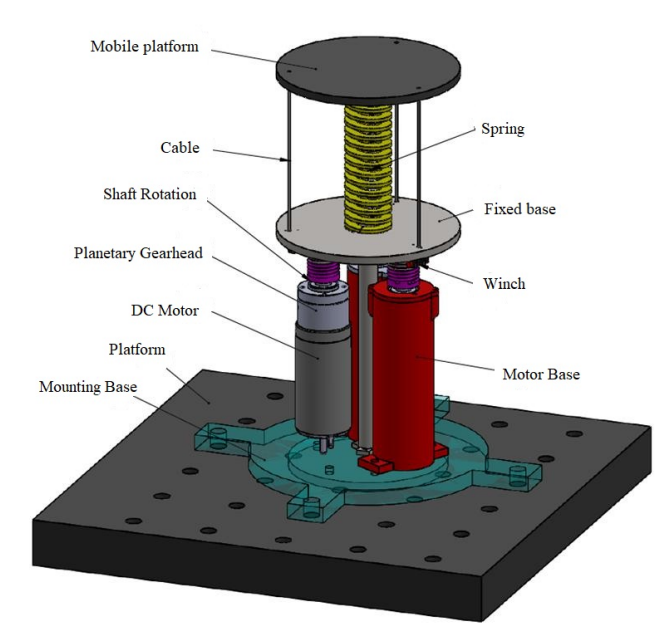

Figure 1: Prototype functional scheme.

coordinate frame oxyz is attached to the moving platform and the y-axis is along $O B_{1}$. The moving platform is driven by three cables and the connection points are $\overrightarrow{o B}=\left(B_{1} ; B_{2} ; B_{3}\right)$; the other end of each cable connects to a roller driven by a motor and the cables pass through the fixed base at points $\overrightarrow{O A}=\left(A_{1} ; A_{2} ; A_{3}\right)$. We will denote the force value along the cable as $\mathrm{T}_{i}$ and the cable length between $A_{i}$ and $B_{i}$ as $l_{i}$.

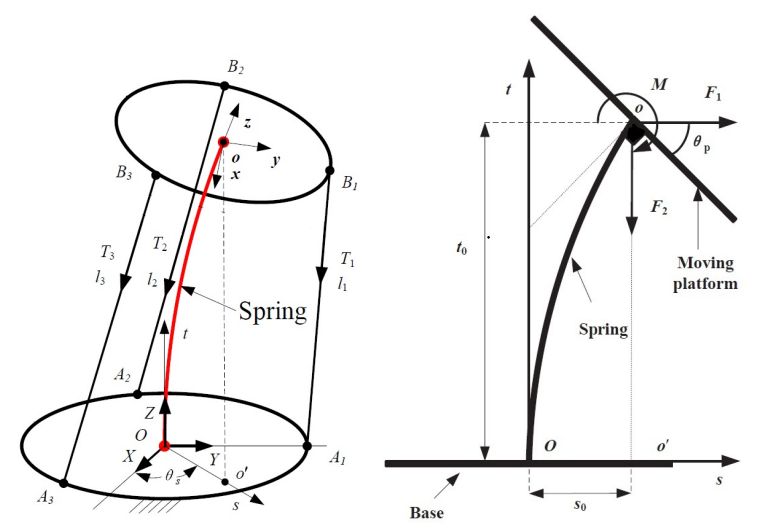

(a) CDMP model. (b) Lateral bending of the spring.

Figure 2: Robot neck diagrams

In the plane formed by $O, o$ and $o^{\prime}$, with $o^{\prime}$ the projection of $o$ to the fixed base, a planar body frame $O s t$ is attached to the spring, as show in Fig. 2(a). The origin is the same as the base coordinate frame $O X Z$, the t-axis is the same as the $\mathrm{Z}$-axis and the s-axis is along $O o^{\prime}$. As shown in Fig. 2(a) and Fig. 2(b), the configuration of the moving platform is defined by four parameters: $\theta_{s}$ is the angle between the s-axis and the $\mathrm{X}$-axis; $\theta_{p}$ is the angle between the fixed base plane and the moving platform plane; $t_{0}$ is the vertical length of the bending spring; $s_{o}$ is lateral translation of the bending spring. There are three parameters 
independent from each other, considering $s_{o}$ as the dependent parameter. In other words, once $\theta_{s}, \theta_{p}$ and $t_{0}$ are given, $s_{o}$ can be solved. In this case, $s_{o}$ is considered as a parasitic movement [24] that can be determined by the other three parameters.

The homogeneous transformation matrix (1) represents the projection from frame oxyz to $O X Y Z$.

$$
{ }^{O} T_{o^{\prime}}=\left[\begin{array}{cc}
{ }^{O} R_{o^{\prime}} & P_{o} \\
0 & 1
\end{array}\right]
$$

where $P_{o}$ is the position vector of point $o$ with respect to the base coordinate frame, $P_{o}=$ $\left[\begin{array}{ccc}s_{o} \cos \theta_{s} & s_{o} \sin \theta_{s} & t_{0}\end{array}\right]^{T}$, and ${ }^{O} R_{o^{\prime}}\left(\theta_{s}, \theta_{p}\right)$ is the rotational matrix that describes the orientation of the moving platform using the Euler angles with orientation $Z Y Z$.

The inverse position kinematics problem is to calculate the cable lengths $L=\left[\begin{array}{lll}L_{1}, & L_{2}, & L_{3}\end{array}\right]^{T}$ given the desired moving platform posture $x=\left[\begin{array}{lll}\theta_{s} & \theta_{p} & t_{0}\end{array}\right]^{T}$.

The cable lengths are calculated by:

$$
L_{i}=\left\|{ }^{O} T_{o^{\prime}} \overrightarrow{o B_{i}}-\overrightarrow{O A_{i}}\right\| \quad(i=1,2,3)
$$

However, $s_{o}$ cannot be an arbitrary number. We transform all the cable forces to two perpendicular forces $F_{1}$ and $F_{2}$ in the bending plane $O s t$, and a moment $M$ perpendicular to the plane at the spring's top center, as shown in Fig. 2(b). The mass of the moving platform $m$ is taken as a mass point at the spring's top center. The equilibrium conditions for force and torque at the moving platform, as shown in Fig. 2(b), are as follows:

$$
\begin{gathered}
\sum_{i=1}^{3}{ }^{O} T_{i}+F=0 \\
\sum_{i=1}^{3}{ }^{O} r_{i} \times{ }^{O} T_{i}+M=0
\end{gathered}
$$

where

$$
\begin{aligned}
& { }^{O} T_{i}=T_{i}\left({ }^{O} T_{o^{\prime}} \overrightarrow{O B_{i}}-\overrightarrow{O A_{i}}\right) /\left\|{ }_{T_{o^{\prime}}} \overrightarrow{o B_{i}}-\overrightarrow{O A_{i}}\right\| \\
& { }^{O} r_{i}={ }^{O} R_{o^{\prime}} \cdot \overrightarrow{o B_{i}} \\
& F=\left[\begin{array}{lll}
-F_{1} \cos \theta_{s}, & -F_{1} \sin \theta_{s}, & F_{2}-m g
\end{array}\right]^{T} \\
& M=\left[\begin{array}{lll}
-M \sin \theta_{s}, & M \cos \theta_{s}, & 0
\end{array}\right]^{T}
\end{aligned}
$$

Considering $\beta$ as the flexural rigidity after compression of the spring [25], the lateral bending curve of the spring spine can be taken as the following linear equation [26]:

$$
\beta \frac{d^{2} s}{d t^{2}}=M+F_{2}\left(s_{0}-s\right)+F_{1}\left(t_{0}-t\right)
$$

with initial conditions:

$$
s(0)=0, s^{\prime}(0)=0, s\left(t_{0}\right)=s_{0} s^{\prime}\left(t_{0}\right)=\tan \theta_{p}
$$

where $s^{\prime}=d s / d t$ and $\beta=\beta_{0} L / L_{0}$. Using the spring bending equation (5) and (6), $s_{O}$ can be obtained; therefore, the inverse position problem can be solved.

Reverse kinematics and static analysis are implemented in Matlab. Additionally, the inertia $I$ and bending constant $\beta_{o}$ of the spring must be calculated with the following equations:

$$
I=\frac{\pi d^{4}}{64} ; \quad \beta_{o}=\frac{2 E G I L_{0}}{\pi N_{a} \frac{d}{2}(E+2 G)}
$$

Table 1: Parameters of selected spring

\begin{tabular}{|c|c|c|c|c|c|c|}
\hline$\epsilon[\mathrm{m}]$ & $L_{o}[\mathrm{~m}]$ & $\mathrm{d}[\mathrm{m}]$ & $\mathrm{Na}$ & $\mathrm{F}[\mathrm{N}]$ & $\mathrm{G}[\mathrm{GPa}]$ & $\mathrm{E}[\mathrm{GPa}]$ \\
\hline 0.001 & 0.1 & 0.003 & 15 & 9.8 & 80 & 200 \\
\hline
\end{tabular}

whose constants values are given in Table 1. The implementation is performed with a fixed $t_{o}=$ $0.085 \mathrm{~m}$, varying $\theta_{p}$ from $0^{\circ}$ to $40^{\circ}$ and $\theta_{s}$ from $0^{\circ}$ to $360^{\circ}$. We can obtain the results shown in Fig. 3, where the cable lengths are located in the z-axis.

In Fig. 3, it is observed that while $\theta_{p}$ is large, the variation in cable length is also large. This is because the more the mobile base is tilted, the greater amount of force will be required.
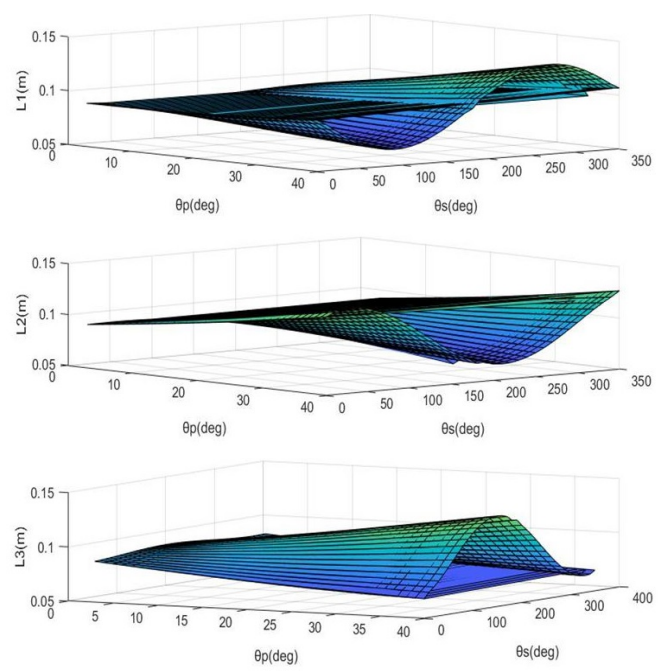

Figure 3: Inverse position kinematics.

\section{Control Problem}

The architecture of the control system for the soft neck is shown in Fig. 4, implemented in Simulink 
Matlab. The scheme has (left to right) input signals, reverse kinematics block, a linear to angular displacement function (to convert cables length into motor position references) for each of the three motors, a PD block and FPD block containing the PD and FPD controllers connected to the transfer function of the motors in closed loop (for the sake of controllers comparison), and finally, an scope to represent the temporal response of the system.

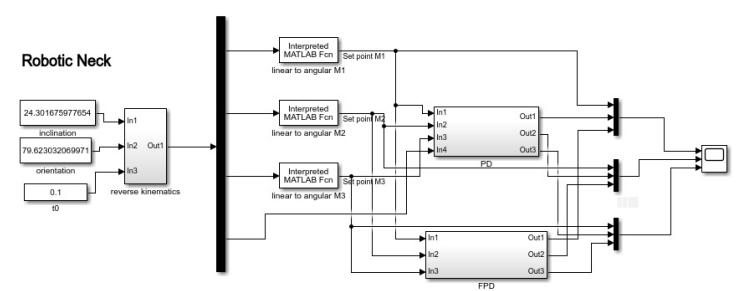

Figure 4: Simulink block diagram.

Each motor will be modeled as a first forder system, having velocity target input and angular joint velocity output, with a gain of 1 , because the motor is commanded directly from the intelligent motor driver. If the encoder is considered, the system output will be position, while the input will still be velocity. In this case, the control variable will be the position, and an integrator must be added to the transfer function, and the final system will be the one in (8). See the response to a unitary step and the Bode diagram for that system in Fig. 5 . Note that phase margin $\phi_{m}=65^{\circ}$, and crossover frequency is $\omega_{c}=30[\mathrm{rad} / \mathrm{s}]$.

$$
G(s)=\frac{1}{(0.1 s+1) s}
$$
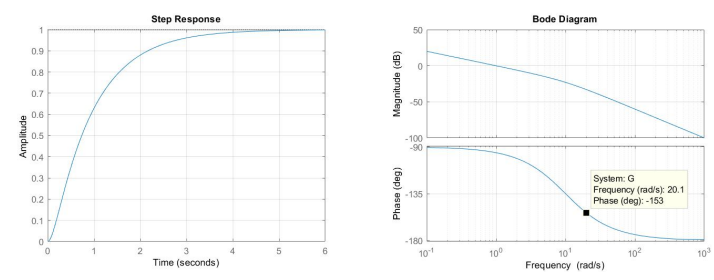

Figure 5: Step response of the motor (left). Bode diagram of the motor (right).

Having an integrator in the plant, the best option is to avoid another integrator in the controller, what leads to a proportional derivative approach.

\subsection{PD Control}

The PD controller is given by

$$
C(s)=K_{p}\left(1+T_{d} s\right)
$$

The transfer function of the open loop system is

$$
C(s) G(s)=\frac{K_{p}\left(1+T_{d} s\right)}{(0.1 s+1) s}
$$

From the basic definitions of phase margin $\phi_{m}$ and crossover frequency $\omega_{c}[27]$, the following set of equations are obtained:

$$
\begin{gathered}
0=\arg \left[G\left(j \omega_{c}\right) C\left(j \omega_{c}\right)\right]+\pi-\phi_{m} \\
0=\left|G\left(j \omega_{c}\right) C\left(j \omega_{c}\right)\right|-1
\end{gathered}
$$

To assign the appropriate value to $K_{p}$ and $T_{d}$, we solve equations (11) and (12) using fsolve function of Matlab (used for solving sets of nonlinear equations [29]). Considering as specifications $\omega_{c}=30$ $[\mathrm{rad} / \mathrm{s}]$ and $\phi_{m}=65^{\circ}$, the resulting controller parameter are $K_{p}=65.22$ and $T_{d}=0.03521$. The Bode diagram of the open loop system with this controller and the step response are shown in Fig. 6 , where it is clearly seen that the specifications are fulfilled.
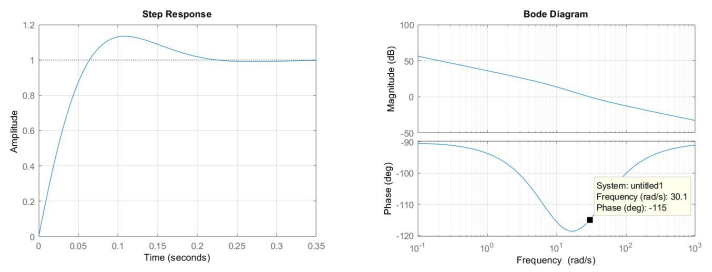

Figure 6: Step response (left), Bode diagram (rigth) for PD Control

\subsection{FPD TUNING: $I S O_{\omega}$ METHOD}

Because of the elastic nature of the system, the motors will be affected by different loads for the different positions. In addition, the neck will accept different payloads attached to the tip, which adds uncertainties to the model and ask for a robust control system. According to this, the control specifications to be met can be summarized as:

- Robustness to mass changes on the tip at a nominal crossover frequency $\omega_{c}$

- Robustness to model uncertainties

The way to meet the first specification is by getting the phase of the open loop system to be flat at $\omega_{c}$ so as the system will be robust to gain changes, as demonstrated in [28]. In this system, the gain of the model is mainly affected by the payloads of the device, as proved in [29]. Therefore, meeting 
this specification will make the system robust to changes in the load.

Here we propose a novel tuning method for an FPD controller of the form in (13) so that the above specifications are met:

$$
C(s)=k_{p}+\tau_{d} s^{\mu}
$$

where $k_{p}$ is the proportional gain, $\tau_{d}$ is the derivative gain, and $\mu$ is the fractional order of the derivative.

A new approach based on the cancellation of the phase slope at a frequency chosen in the Bode diagram will be used for tuning. After phase cancellation, the controlled system will have flat phase around the desired frequency $\omega_{c}$, achieving robustness to the changes in motor loads.

In this kind of controller, the phase slope can be computed as:

$m=\frac{d}{d \log _{10}(\omega)} \arctan \left(\frac{\sin (\mu \pi / 2)}{\frac{1}{k_{d} 10^{\log _{10}(\omega)^{\mu}}}+\cos (\mu \pi / 2)}\right)$

and simplified with the change of variable $\log _{10}(\omega)=x$, it results:

$$
m=\frac{d}{d x} \arctan \left(\frac{\sin (\mu \pi / 2)}{\frac{1}{k_{d} 10^{x \mu}}+\cos (\mu \pi / 2)}\right)
$$

In (14), all the slopes for all the frequencies $(\omega)$ and parameters $\left(k_{d}, \mu\right)$ are defined, but for the sake of simplicity, only one case will be considered. Selecting the frequency $\omega^{\mu}=1 / k_{d}$ (greatest slope) offers a great simplification, and equation (14) becomes:

$$
m_{c}=\tan (\mu \pi / 4) \mu \log (10) / 2
$$

Plotting the values of $m_{c}$ in front of $\mu$, it is possible to find $\mu$ from the slope. All values of $m_{c}$ are computed for $\mu=(0,1.6)$ obtaining the curve in Fig. 7 , for the specific case $\omega^{\mu}=1 / k_{d}$.

Having the exponent $\mu$, it is needed to make the controller fit the frequency of interest. Parameters $k_{d}$ and $\mu$ have an effect on the crossover frequency at which the phase slope cancellation occurs in the Bode diagram.

Plotting all the values of $\omega$ in front of $\mu$ and $k_{d}$, for the case of $\omega^{\mu}=1 / k_{d}$ to keep consistency, we obtain Fig. 8. It is a computer demanding task, but once done, the graph can be easily used by graphical interpolation.

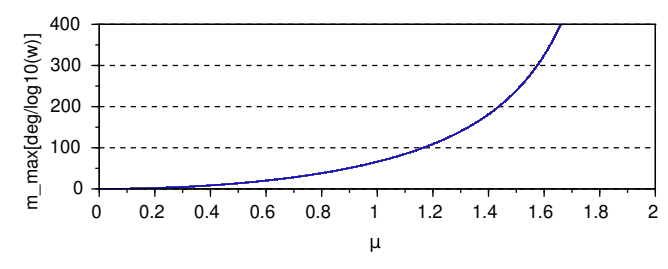

Figure 7: Phase slope plot in the Bode diagram at the frequency at which point $\phi(\omega)=\mu \cdot 45^{\circ}$, as $\mu$ changes.

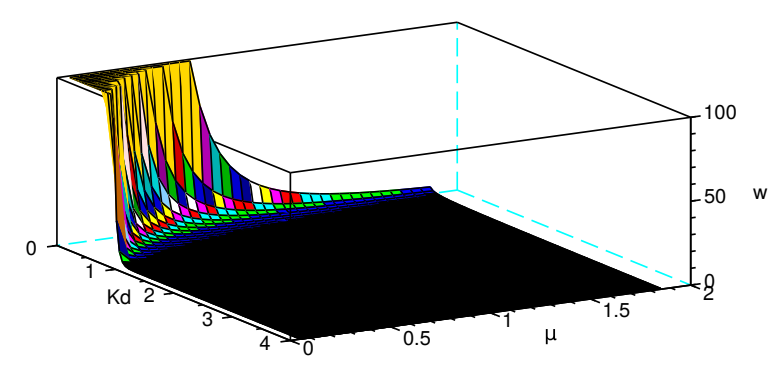

Figure 8: Surface of controller parameters, showing $\omega$ as a funtion of $\mu$ and $k_{d}$.

Once the surface is computed, an $I S O_{\omega}$ graph can be obtained as shown in Fig. 9, which allows, given a value of $\omega$ within the range of values chosen during the graph computing, and a value of $\mu$ that cancels the slope, to select a value of $k_{d}$ that sets the controller frequency in order to perform the cancellation at the correct place $\left(\omega_{c}\right)$.

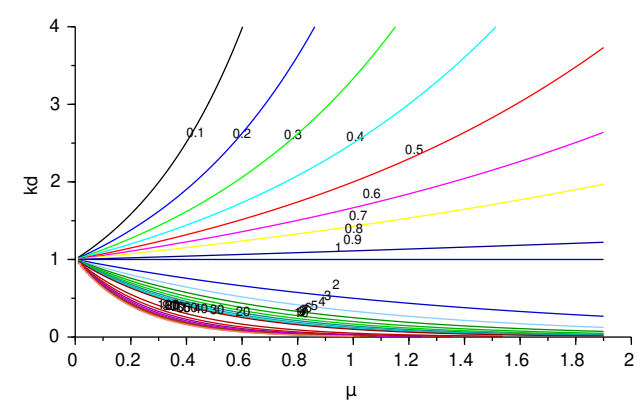

Figure 9: Parameter tuning plot, showing isow curves as a funtion of $\mu$ and $k_{d}$.

After that, $k_{p}$ will be used to set the open loop system magnitude to $0[\mathrm{~dB}]$ at $\omega_{c}$, finishing this way the tuning procedure.

Now that the method has been presented, it will be used for the controller tuning, summarized in the following steps:

-Choose $\omega_{c}$

As in the previous tuning, $\omega_{c}=30[\mathrm{rad} / \mathrm{s}]$

-Find the system slope at $\omega_{c}$ 
Checking the phase curve in Fig. 5, the slope at $\omega_{c}$ is $m=-40\left[\mathrm{deg} / \log _{10}(\omega)\right]$.

-Find $\mu$

Using Fig. 7, we find an exponent $\mu$ corresponding to that value of $m$.

-Look for $k_{d}$

For the $I S O_{\omega}$ curve $\omega_{c}=30[\mathrm{rad} / \mathrm{s}]$ in Fig. 9, and $\mu=0.8$, there is only one possible value of $k_{d}=0.07$.

-Set $0[\mathrm{~dB}]$ at $\omega_{c}$

The proportional constant is set for the open loop system magnitude to be $0[\mathrm{~dB}]$ for $\omega_{c}=30[\mathrm{rad} / \mathrm{s}]$, then:

$$
k_{p}=\frac{1}{\left|\bar{C}\left(j \omega_{c}\right) G\left(j \omega_{c}\right)\right|}=59
$$

-Controller parameters

Once all the constants are known, the controller results

$$
\begin{gathered}
C(s)=k_{p}+\tau_{d} s^{\mu} \\
C(s)=59\left(1+0.07 s^{0.8}\right)=59+4.13 s^{0.8}
\end{gathered}
$$

The result is shown in Fig. 10. A flat slope at a crossover frequency is shown, according to the requirements. The step response of the controlled system is also shown.

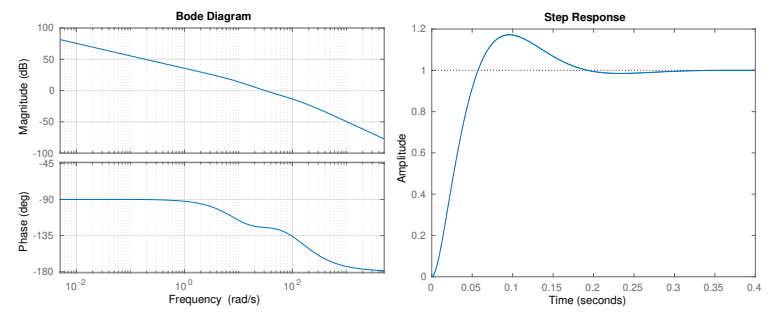

Figure 10: Bode diagram and step response for FPD controller based on the $I S O_{\omega}$ method.

\section{Simulation results}

Performing the robotic neck simulation with the PD and FPD control system for different mass gains $\mathrm{Gw}=(1,0.6,0.3)$, the robustness of the designed controllers is checked.

In the Fig. 11 it can be seen that the PD controller has different overshoots for changing masses while the FPD controller has a constant overshoot (iso-damping) and presents a very robust performance. The introduction of the fractional order $\mu$ in the controller and the application of the tuning method proposed enhance the performance of the FPD-based system in comparison with the PD-based system.
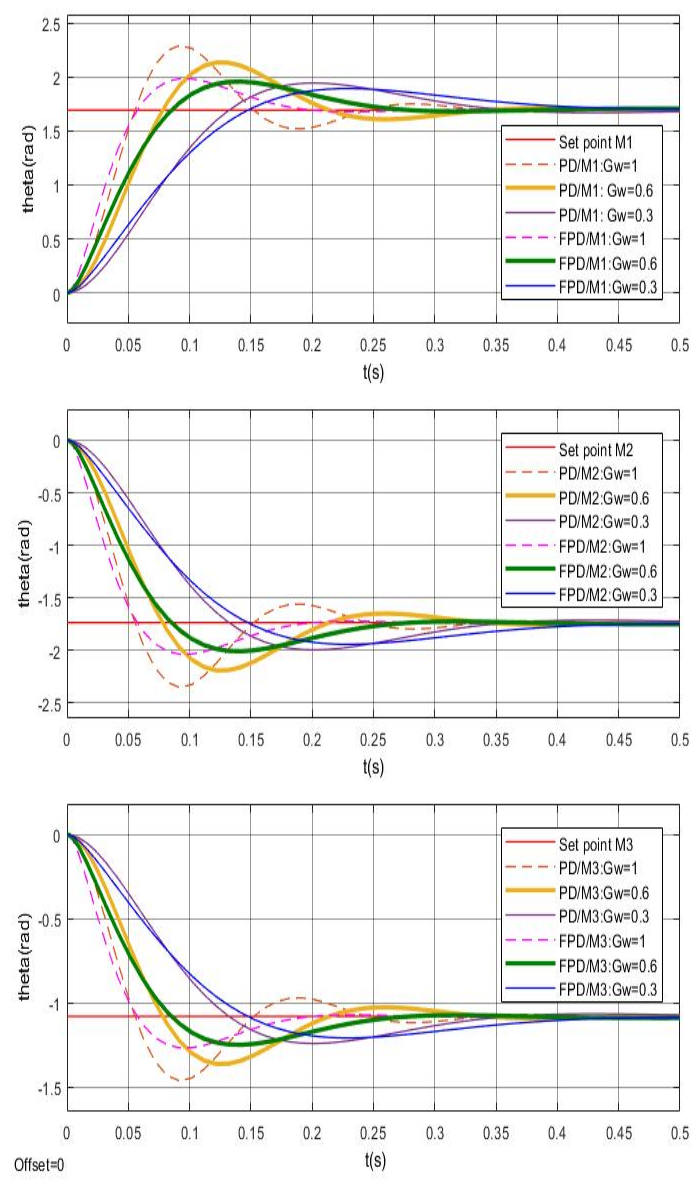

Figure 11: Comparison of the responses of all the controllers (PD and FPD) for different masses at the tip. Step input of $\theta_{p}=15^{\circ}$ and $\theta_{s}=80^{\circ}$.

\section{Conclusions}

This paper has presented the design of a soft robotic neck with two DOF providing pitch and roll movements. The constraint is a maximum inclination of $40^{\circ}$. The mechanical and electrical design of the prototype have been addressed taking this constraint into account. The mechanism uses a coil compression spring to simulate the cervical vertebrae and cables that act as muscles. The mathematical model of the neck has been presented and the theoretical static analysis of the mechanism has been performed.

A novel tuning method for a fractional order PD controller (FPD) has been proposed with applications to the control of the soft robotic neck. This method is based on the graphical solution of the controller parameters, avoiding the resolution of nonlinear equations sets and allowing to solve the 
control problem in a very intuitive and direct way. The resulting FPD controller outperforms the PDbased performance of the system in terms of robustness.

In the future, the proposed soft neck will be built and tested, and the tuning method will be extended to fractional order PID controllers.

\section{Acknowlegments}

The research leading to these results has received funding from the HUMASOFT project, with reference DPI2016-75330-P, funded by the Spanish Ministry of Economy and Competitiveness.

\section{References}

[1] H. Hirukawa, F. Kanehiro, K. Kaneko, S. Kajita, K. Fujiwara, Y. Kawai, F. Tomita, S. Hirai, K. Tanie, T. Isozumi, et al., "Humanoid robotics platforms developed in hrp," Robotics and Autonomous Systems, vol. 48, no. 4, pp. 165-175, 2004.

[2] Y. Sakagami, R. Watanabe, C. Aoyama, S. Matsunaga, N. Higaki, and K. Fujimura, "The intelligent asimo: System overview and integration," in Intelligent Robots and Systems, 2002. IEEE/RSJ International Conference on, vol. 3. IEEE, 2002, pp. 2478-2483.

[3] Y. Tadesse, K. Subbarao, and S. Priya, "Realizing a humanoid neck with serial chain four-bar mechanism," Journal of Intelligent Material Systems and Structures, vol. 21, no. 12, pp. 1169-1191, 2010.

[4] J. Han, S. Zeng, K. Tham, M. Badgero, and J. Weng, "Dav: A humanoid robot platform for autonomous mental development," in Development and learning, 2002. Proceedings. The 2nd international conference on. IEEE, 2002, pp. 73-81.

[5] R. Beira, M. Lopes, M. Praça, J. SantosVictor, A. Bernardino, G. Metta, F. Becchi, and R. Saltarén, "Design of the robot-cub (icub) head," in Robotics and Automation, 2006. ICRA 2006. Proceedings 2006 IEEE International Conference on. IEEE, 2006, pp. 94-100.

[6] T. Hashimoto, S. Hitramatsu, T. Tsuji, and H. Kobayashi, "Development of the face robot saya for rich facial expressions," in SICE-ICASE, 2006. International Joint Conference. IEEE, 2006, pp. 5423-5428.
[7] I. Podlubny, "Fractional-order systems and $\mathrm{pi} / \sup / \mathrm{spl}$ lambda//d/sup /spl mu//controllers," IEEE Transactions on Automatic Control, vol. 44, no. 1, pp. 208-214, Jan 1999.

[8] C. Monje, B. Vinagre, V. Feliu, and Y. Chen, "Tuning and auto-tuning of fractional order controllers for industry applications," Control Engineering Practice, vol. 16, no. 7, pp. 798 - 812, 2008.

[9] A. Rastogi and P. Tiwari, "Optimal tuning of fractional order pid controller for dc motor speed control using particle swarm optimization," 2013.

[10] K. Ranjbaran and M. Tabatabaei, "Fractional order [pi], [pd] and [pi][pd] controller design using bode's integrals," International Journal of Dynamics and Control, vol. 6, no. 1, pp. 200-212, Mar 2018. [Online]. Available: https://doi.org/10.1007/s40435016-0301-7

[11] L. Dorcak, "Numerical models for the simulation of the fractional-order control systems," ArXiv Mathematics e-prints, Apr. 2002.

[12] I. Petras, "Fractional order feedback control of a dc motor," Journal of Electrical Engineering, vol. 60, no. 3, pp. 117-128, 2009.

[13] T. Qingshun, W. Chunfu, Y. Yuanhui, L. Guodong, and Z. Fengyu, "Design and implementation of fractional order controller for service robots," International Journal of Control and Automation, vol. 8, no. 5, pp. 209-220, 2015.

[14] C. A. Monje, Y. Chen, B. M. Vinagre, D. Xue, and V. Feliu, Fractional-order Proportional Derivative Controller Tuning for Motion Systems. London: Springer London, 2010, pp. 107-120.

[15] K. Khandani and A. A. Jalali, "Robust fractional order control of a dc motor based on particle swarm optimization," in $M E M S$, NANO and Smart Systems, ser. Advanced Materials Research, vol. 403. Trans Tech Publications, 2 2012, pp. 5030-5037.

[16] M. F. Silva and J. A. T. Machado, "Fractional order pd joint control of legged robots," 2006.

[17] D. Karaboga and B. Basturk, "A powerful and efficient algorithm for numerical function optimization: artificial bee colony (abc) algorithm," Journal of global optimization, vol. 39, no. 3, pp. 459-471, 2007. 
[18] A. Rajasekhar, P. Kunathi, A. Abraham, and M. Pant, "Fractinal order speed control of dc motor using levy mutated artificial bee colony algorithm," in 2011 World Congress on Information and Communication Technologies, Dec 2011, pp. 7-13.

[19] V. Haji Haji and C. A. Monje, "Fractionalorder pid control of a chopper-fed dc motor drive using a novel firefly algorithm with dynamic control mechanism," Soft Computing, Jun 2017. [Online]. Available: https://doi.org/10.1007/s00500-017-2677-5

[20] F. MartÃn, C. A. Monje, L. Moreno, and C. Balaguer, "De-based tuning of $\mathrm{pi} \lambda \mathrm{d} \mu$ controllers," ISA Transactions, vol. 59, pp. 398 $-407,2015$.

[21] S. Al-Ratrout, A. Saleem, and H. Soliman, "Optimization methods in fractional order control of electric drives: A comparative study," in 2015 10th International Symposium on Mechatronics and its Applications (ISMA), Dec 2015, pp. 1-6.

[22] S. Behzadipour and A. Khajepour, "Cablebased robot manipulators with translational degrees of freedom," in Industrial Robotics: Theory, Modelling and Control. InTech, 2006, pp. 221-236.

[23] S. Alfayad, M. El Asswad, A. Abdellatif, F. B. Ouezdou, A. Blanchard, N. Beaussé, and P. Gaussier, "Hydroïd humanoid robot head with perception and emotion capabilities: Modeling, design, and experimental results," Frontiers in Robotics and AI, vol. 3, p. $15,2016$.

[24] J. Carretero, R. Podhorodeski, M. Nahon, and C. M. Gosselin, "Kinematic analysis and optimization of a new three degree-offreedom spatial parallel manipulator," Journal of mechanical design, vol. 122, no. 1, pp. $17-24,2000$.

[25] S. Timoshenko, Theory of elastic stability, 1st ed. New York McGraw-Hill Book Company, inc, 1936, bibliographical foot-notes. [Online]. Available: http://openlibrary.org/books/OL24757780M

[26] B. Gao, H. Song, L. Sun, and Y. Tang, "Inverse kinematics and workspace analysis of a bio-inspired flexible parallel robot," in 2013 IEEE International Conference on Cyber Technology in Automation, Control and Intelligent Systems, May 2013, pp. 138-143.
[27] W. K. Ho, C. C. Hang, and L. S. Cao, "Tuning of pid controllers based on gain and phase margin specifications," Automatica, vol. 31, no. 3, pp. 497-502, 1995.

[28] Y. Chen and K. L. Moore, "Relay feedback tuning of robust pid controllers with iso-damping property," Trans. Sys. Man Cyber. Part B, vol. 35, no. 1, pp. 23-31, Feb. 2005. [Online]. Available: http://dx.doi.org/10.1109/TSMCB.2004.837950

[29] V. Feliu, B. M. Vinagre, and C. A. Monje, Fractional-order Control of a Flexible Manipulator. Dordrecht: Springer Netherlands, 2007, pp. 449-462.

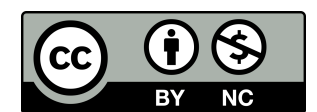

(C) 2018 by the authors. Submitted for possible open access publication under the terms and conditions of the Creative Commons Attribution CC-BY-NC 3.0 license (http://creativecommons.org/licenses/by-nc/3.0/). 